Boletín de la Sociedad Botánica de México 49: 19-30, 1989

DOI: 10.17129/botsci.1363

Bol. Soc. Bot. México 49:19-30(1989)

\title{
Aspectos ecofisiológicos de las micorrizas
}

\author{
MARIA VALDES ${ }^{1}$
}

RESUMEN. La micorriza es la parte de las raíces infectadas por ciertos hongos del suelo. Este tipo de asociación está presente en la mayoría de las especies vegetales. Hay diferentes tipos de micorrizas, las de mayor đistribución en la naturaleza son la Ectomicorriza (EM) y la Micorriza Vesículo-Arbuscular (MVA), a las que nos referiremos en esta breve revisión.

La EM forma un manto fúngico compacto sobre la superficie radicular y las hifas penetran intercelularmente; la MVA forma una red laxa de hifas en el suelo alrededor de la raíz y las mismas penetran en el interior de las células corticales.

Las micorrizas incrementan la toma de nutrientes y de agua por las plantas, sobretodo incrementan el transporte activo hacia la planta de iones poco móviles como el P, por lo tanto estimulan considerablemente el crecimiento de las mismas. Este incremento se lleva a cabo ya que las hifas de los hongos se extienden enormemente alrededor de la raíz formando una amplia área de absorción, es decir, las micorrizas acortan la distancia que deben pasar los nutrientes a través del suelo hacia la raíz además de poner a la disposición de la planta mayor volumen de suelo para absorber nutrientes.

La mayoría de las plantas muestran respuestas fisiológicas a la colonización for los hongos según el nivel de fertilidad del suelo y según su dependencia micorrícica. Generalmente una alta fertilidad del suelo conduce a una colonización mínima, por lo que en suelos de agricultura intensiva la micorriza se extingue. La dependencia micorrícica varía de planta a planta y está en relación a la cantidad de pelos radiculares, siendo mas dependientes de los hongos micorrícicos aquellas plantas de raíces gruesas no ramificadas y con escasos pelos.

Casi todos los suelos contienen diferentes propágulos de hongos micorrícicos y su distribución varía con el clima, ambiente edáfico y uso del suelo. No todos los hongos aportan el mismo grado de beneficio a las plantas.

ABSTRACT. Mycorrhiza is the part of the roots infected with particular soil fungi. This type of association is formed by most of the plants. There are several types of mycorrhizae; this short review is concerned only with Ectomycorrhiza (EM) and the Vesicular-Arbuscular Mycorrhiza (VAM). These two types are the most common in nature.

${ }^{1}$ Escuela Nacional de Ciencias Biológicas, Instituto Politecnico Nacional, 11410, México, D.F. 
EM has a compact fungus mantle over the root surface and intercellular hyphae in the cortex; the VAM has a loose network of hyphae in the soil surrounding the root and hyphal growth within the cortical cells.

Mycorrhizas increase nutrient uptake and hence plant growth. Since mycorrhizas are surrounded by an extensive hyphal network than may extend into the soil, this network represents a greater surface area, in other words, mycorrhizas shorten the distance that nutrients must diffuse through the soil to the root and their hyphae increase the volume of soil available to the plant for nutrient uptake.

Physiological responses to root colonization with mycorrhizal fungi by most of the plants are dependent on the level of soil fertility and on the degree of mycorrhizal dependency of the plant. Soils having a high fartility have mostly a poor colonization, hence, for plant growth to respond to inoculation, soils must have a low fertility. Mycorrhizal dependency can be very different among plant species; plants with short root hairs are more dependant on mycorrhizal fungi.

Most soils contain mycorrhizal fungi and their distribution varies with climatic, edaphic environment and land use. There are differences in effectiveness in colonization and in enhanced nutrient uptake among the fungi.

\section{INTRODUCCION}

No cabe duda que es alentadora la perspectiva de usar micorrizas en la agricultura para reducir la aplicación de fertilizantes, especialmente fósforo, o bien de obtener árboles sanos y vigorosos capaces de prosperar en áreas a reforestar muy adversas.

¿Qué es la micorriza? Este término designa la asociación de hifas fúngicas de ciertos hongos a los órganos subterráneos de las plantas superiores. Esta asociación culmina con la formación de un órgano nuevo con morfología y fisiología propias.

Estas asociaciones prevalecen en la mayoría de las especies vegetales, es decir, la micorriza forma parte de la raíz, en la misma forma que el cloroplasto forma parte de la hoja.

\section{TIPOS DE MICORRIZA}

Las micorrizas se han agrupado en diferentes tipos basadas en las estructuras hongo-raíz. En este escrito voy a referirme a los dos grupos de mayor distribución en la naturaleza: ectomicorriza y micorriza vesículo-arbuscular (VA).

En la ectomicorriza el hongo forma una estructura llamada manto que encierra al pelo absorbente; las hifas penetran intercelularmente formando una red conocida como red de Hartig. En la endomicorriza o micorriza VA no existen cambios morfológicos visibles a simple vista en la estructura externa de la raíz, pero las hifas penetran en la células corticales formando diferentes estructuras que pueden ser vesículas, arbúsculos o ambos. Las hifas de las micorrizas salen en grandes cantidades hacia el exterior 
formando una gran área de absorción y mediando el transporte de minerales y de otros materiales del suelo hacia la planta.

La micorriza VA está presente en la mayoría de las plantas cultivadas, en la mayoría de los árboles frutales y en la mayoría de los árboles forestales; las plantas no cultivadas también forman MVA en su ambiente natural. El principal valor de este tipo de micorriza proviene de su capacidad de proveer a la planta con mas nutrimentos, sobretodo P, Cu y Zn. Estos iones son muy poco móviles y forman alrededor de los pelos absorbentes zonas de agotamiento.

Otros beneficios que pueden recibir las plantas con micorriza VA son: reducción de efectos de patógenos fúngicos, supresión de efectos de nemátodos parásitos, incremento en absorción de agua e incremento de tolerancia a suelos salinos y a sequía.

La ectomicorriza se presenta en árboles de la Fam. Pinaceae (pino, abeto), Fagaceae (encino), Betulaceae (aile) y Salicaceae (chopo), entre otras. La ectomicorriza beneficia a la planta en diferentes formas: incrementa la longevidad de los pelos radiculares, incrementa la tasa de absorción de elementos mayores y menores del suelo, aumenta la tolerancia a las toxinas del suelo, a altas y bajas temperaturas y a pH adverso y confiere resistencia a las raíces hacia algunos patógenos.

\section{EFECTOS DE LA MICORRIZA EN LA NUTRICION MINERAL Y EN EL USO DEL AGUA DE} LAS PLANTAS

El gran interés despertado en los últimos años por el estudio de las micorrizas se justifica plenamente pues inducen en las plantas un estímulo muy importante en su crecimiento, particularmente por la aportación de P. Tanto las plantas micorrizadas como las no micorrizadas toman el P de la solución del suelo. La concentración del mismo en el suelo es baja (menos de $1 \mu \mathrm{M}$ ) por lo que el flujo de masa del P es insuficiente para abastecer a la planta con este elemento. Se ha estimado que el flujo de masa contribuye en algunas plantas con sólo del 3 al 13\% de la captación de P (Itoh y Barber, 1983).

La difusión es el paso limitante y el camino más importante para el movimiento de los iones hacia la raíz, en el caso del P es particularmente lenta en el suelo (aproximadamente $10^{-9} \mathrm{~cm}^{2} / \mathrm{seg}$ ). Se sabe, a través de estudios de la tasa de movimiento de nutrientes hacia las raíces de las plantas y de tasas de absorción de los mismos por las raíces, que la captación de N, P y K está limitada por la tasa de difusión de cada nutriente a través del suelo y no por la capacidad de la raíz para absorberlos a partir de las bajas concentraciones de los mismos en la solución del suelo (Nye, 1977). Siendo ésta la limitante y dado que las hifas de las micorrizas se extienden, enormemente de la raíz micorrizada hacia el suelo alrededor de la misma, incrementan efectivamente igual que los pelos absorbentes, el área de absorción y consecuentemente la captación de nutrientes, básicamente por acortamiento de la distancia que deben pasar los nutrientes a través del suelo hacia la raíz. También incrementan esta captación por el mayor volumen de suelo disponible a la planta. 
Se ha mostrado que las hifas de la MVA pueden interceptar fósforo marcado colocado a una distancia de 3-6 cm de la raíz micorrizada, mientras que el mismo permanece inaccesible a las raíces no micorrizadas (Rhodes y Gerdemann, 1975). En el caso de las ectomicorrizas, esta distancia es mayor.

Aún cuando la difusión no limite la absorción, también se han observado diferencias entre raíces micorrizadas y no micorrizadas en sus tasas de absorción de nutrientes de la solución del suelo. Las raíces micorrizadas absorben más rápido $\mathrm{P}, \mathrm{S}, \mathrm{Zn}$ y otros nutrientes por gramo de raíz que las raíces no micorrizadas (Bowen et al. 1974 y 1975). Posiblemente esto también está relacionado con la mayor área de supeficie/g de las raíces micorrizadas. También son capaces de aumentar la absorción de nutrientes del suelo que se mueven hacia las raíces por flujo de masa, además de aquellos que difunden lentamente a través del suelo. El incremento en la captación de otros elementos, en particular de $\mathrm{K}$, es probablemente una consecuencia del abastecimiento incrementado de P.

Así como hay cambios en el crecimiento de las plantas debidos a la infección por hongos micorrícicos, se ha mostrado con hongos VA que pueden causar cambios en algunos aspectos de las relaciones de agua de la planta hospedera como son la conductividad hidráulica de la raíz, el potencial de agua de la hoja y la resistencia de la hoja. En algunos casos se ha mejorado la resistencia o tolerancia a la sequía.

La mayoría de estos fenómenos son quizás el resultado de una nutrición fosforada mejorada (Safir y Nelsen, 1980), sin embargo se han hecho ensayos que sugieren que las micorrizas pueden mantener el abastecimiento de $\mathbb{P}$ cuando su tasa de difusión está marcadamente restringida por un suelo seco, no siendo así en las raíces no infectadas. Esta hipótesis se ha tornado en modelo matemático (Fitter, 1985) el cual sugiere que en general la importancia de la micorriza en la nutrición fosforada de las plantas se incrementará notablemente al reducirse el contenido de agua del suelo.

\section{FACTORES QUE AFECTAN LA RESPUESTA VEGETAL}

a) Fertilidad del suelo.

La mayoría de las plantas micorrizadas se benefician con la simbiosis y muestran incrementado su crecimiento, su absorción de nutrientes, su fijación de $\mathrm{N}^{2}$ (si también se asocian con Rhizobium o con Frankia), etc. Sin embargo, la mayoría de las plantas muestran estas respuestas fisiológicas a los hongos micorrícicos a niveles bajos de P. (Azcón et al., 1978; Hayman, 1982; Nelsen et al., 1981; Plenchette et al., 1983), una alta fertilización nitrogenada también ha mostrado afectar negativamente la formación de micorriza (Hayman, 1970).

El P ha recibido mayor atención en los estudios de micorrizas por el marcado efecto del nivel del mismo en el desarrollo de las mismas.

El nivel de $P$ en el suelo está relacionado con la colonización radicular por los hongos micorrícicos; al haber un nivel bajo de $\mathrm{P}$, hay un bajo nivel de fosfolípidos en la membrana vegetal que conduce a una mayor exudación radicular que trae como consecuencia una estimulación a la colonización por el endófito (Fig.1). Además, la actividad endofítica 
(estimada tanto por número de esporas como por colonización porcentual) se deprime cuando se adiciona P soluble (Nelsen et al., 1981).

Por otro lado el desarrollo de micorriza a un nivel de $\mathrm{P}$ no limitante para el crecimiento, puede no ser lo suficiente extensivo para estimular la captación de otros nutrientes que están presentes a niveles deficientes para el máximo crecimiento de las plantas.

Generalmente una alta fertilidad del suelo conduce a una colonización MVA mínima, tanto que dificilmente se encuentra este tipo de micorriza en suelos cultivados intensamente. Algunas plantas pueden obtener beneficios de la micorriza VA independientemente del nivel de $\mathrm{P}$ del suelo, a estas plantas se les llama dependientes de los hongos MVA (ej: cebolla, yuca).

La temperatura del suelo y la intensidad luminosa también influyen en la colonización y en la respuesta de la planta. La mayoría de los hongos tienen un óptimo de temperatura para establecer la relación simbiótica y persistencia de la micorriza ya formada (Furlan y Fortin, 1973). El mayor desarrollo micorrícico a intensidades de luz elevadas parece estar relacionado a una actividad fotosintética mayor y consecuentemente un mayor transporte de carbohidratos hacia la raíz; sin embargo, en algunos casos en condiciones sombreadas hay micorrización más extensiva (Furlan y Fortin, 1977).

b) Planta. Dependencia micorrícica.

La respuesta de la planta valorada en cuanto a colonización radicular y crecimiento, varía considerablemente de planta a planta. Algunas plantas no forman micorriza cuando se les inocula con hongos MVA, lo que indica que la planta juega un papel importante en la simbiosis. Sin embargo, como ya se señaló, la mayoría de las plantas forman micorriza (Fig. 2).

Se acepta, en general, y en base a muchos resultados, que las plantas con raíces gruesas, no ramificadas y con escasos pelos (ej. cítricos, cebollas) son más dependientes de la micorriza que aquellas plantas con raíces muy ramificadas con numerosos y largos pelos radiculares. Baylis (1970) mostró que el grado de formación de pelos radiculares en muchas plantas está relacionado inversamente con un incremento en la dependencia hacia la MVA y directamente correlacionado con la capacidad de la planta en absorber $\mathrm{P}$ de los suelos con baja cantidad de $\mathrm{P}$ disponible.

Las plantas altamente dependientes para su producción de altos niveles de fertilización fosforada, en ausencia de éstos, dados sus requerimientos en $\mathrm{P}$, son altamente dependientes de la micorriza. La dependencia micorrícica de una planta está relacionada pues, a la fertilidad del suelo. Esta dependencia también está bajo la influencia de la tasa de crecimiento de la planta, especies de hongo y tipo de suelo.

La dependencia micorrícica se define como "el grado al cual una planta requiere de la condición micorrícica para dar su máximo crecimiento o rendimiento a un nivel dado de fertilidad del suelo" (Gerdeman, 1975). Así, se ha definido este parámetro numéricamente como la diferencia entre el peso seco de una planta micorrizada y una no micorrizada, expresada como porcentaje o bien como la tasa de incremento de masa seca de la planta micorrizada en relación a la masa seca de la planta no micorrizada. Las 


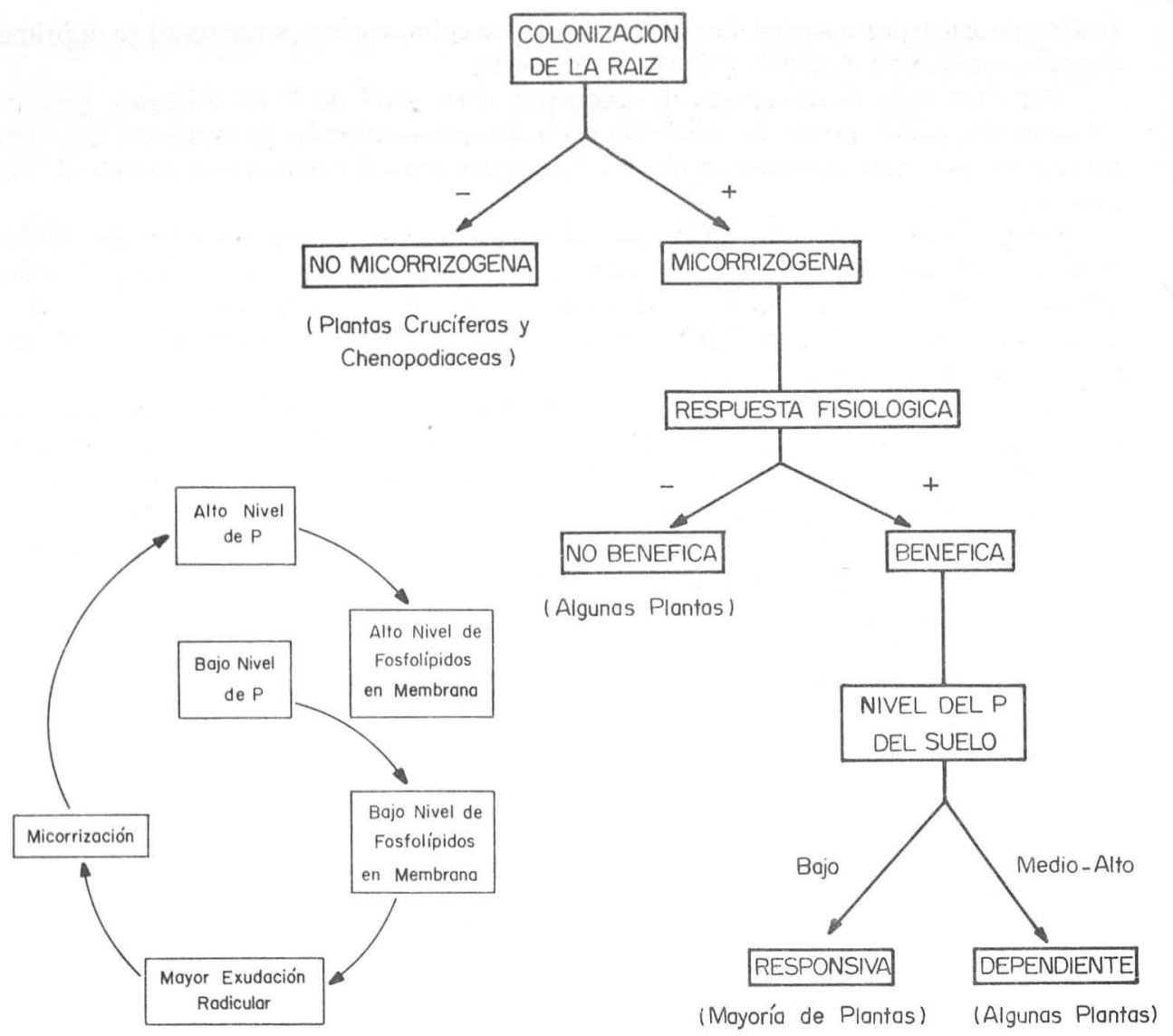

Figura 1.- La colonización radicular por hongos micorrízicos depende en gran medida del nivel de $\mathrm{P}$ en el suelo; con un bajo nivel de $\mathrm{P}$, el nivel de fosfolípidos en las membranas radiculares será también bajo, lo que conduce a una mayor exudación radicular, exudación vital para la reproducción de los propágulos y su colonización.

Figura 2.- Tipos de respuesta hospedera a la inoculación micorrizógena, incluyendo micorrizógena y no micorrizógena (basada en la colonización) benéfica y no benéfica (basada en la respuesta fisiológica de la planta) y responsiva o dependiente (basada en el nivel de P del suelo). Según Miller et al., 1986. 
TABLA 1.- SOBREVIVENCIA Y DESARROLLO DE PINUS PSEUDOSTROBUS INOCULADO CON DIFERENTES HONGOS ECTOMICORRIZICOS DESPUES DE 3 AÑOS DE CRECIMIENTO EN UN SITIO SEVERAMENTE EROSIONADO. ${ }^{1}$

\begin{tabular}{lcccc}
\hline Tratamiento & $\begin{array}{c}\text { Sobrevivencia } \\
\%\end{array}$ & $\begin{array}{c}\text { Altura } \\
(\mathrm{cm})\end{array}$ & $\begin{array}{c}\text { Diámetro } \\
\text { del tallo } \\
(\mathrm{mm})\end{array}$ & $\begin{array}{c}\text { Volúmen } \\
\text { de las } \\
\text { plántulas } \\
\left(\mathrm{cm}^{3 *}\right) 2 / \\
100\end{array}$ \\
& & & & \\
\hline $\begin{array}{l}\text { Pepiota lutea } \\
\text { Pisolithus tinctorius }\end{array}$ & $84 \mathrm{a}$ & & $38.0 \mathrm{a}$ & $1975 \mathrm{a}$ \\
$\begin{array}{l}\text { Laccaria laccata } \\
\text { Testigo }\end{array}$ & $56 \mathrm{c}$ & $96.5 \mathrm{a}$ & $33.0 \mathrm{~b}$ & $1556 \mathrm{a}$ \\
& $50 \mathrm{c} 4$ & $63.0 \mathrm{~b}$ & $20.8 \mathrm{c}$ & $314 \mathrm{~b}$ \\
& $44.5 \mathrm{c}$ & $25.3 \mathrm{c}$ & $367 \mathrm{~b}$ \\
\hline
\end{tabular}

1/ Cada valor es la media de 50 plantas de pino de 3 repeticiones

$2 /$ Volumen de las plántulas $\left(\mathrm{cm}^{3}\right)=$ (Diámetro del tallo) ${ }^{2} \times$ altura

3/ Cada columna seguida por la misma letra no es significativamente diferente a $\mathrm{P}=0.01$. 


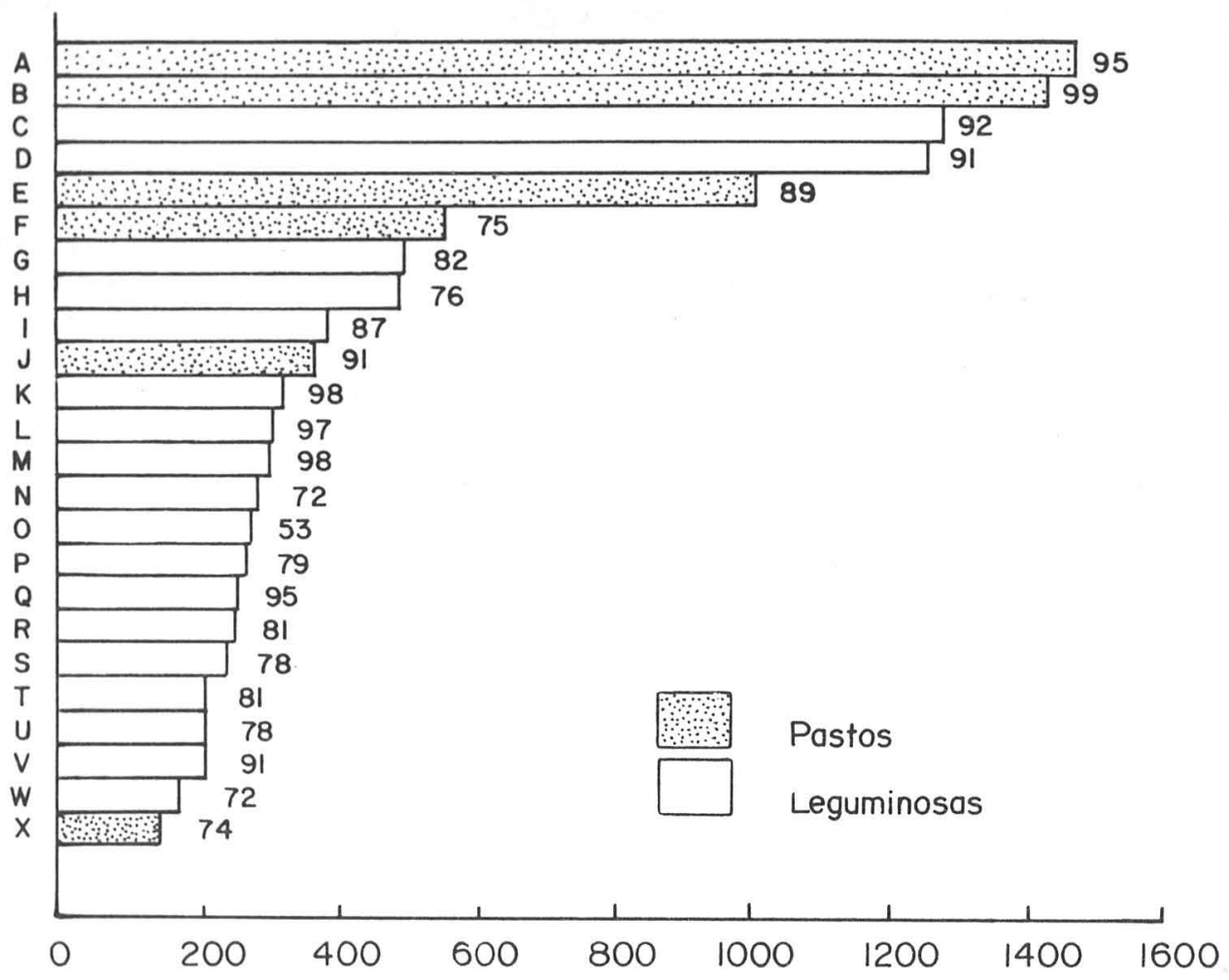

Figura 3.- Dependencia micorrícica de 24 plantas forrajeras determinado por el porcentaje del peso seco de las plantas micorrizadas en relación a las no micorrizadas. Los números en las barras indican el porciento de la micorrización de la raíz (Saif, 1987). Las especies son: 1.- Brachiaria decumbens, 2.- B. brizantha, 3. Zornia glabra, 4.- Z. brasilensis, 5.- Andropogon gayanus, 6.- B. dictyoneura, 7.- Centrosema macrocarpum, 8.- C. brasilianum, 9.- C. pubescens, 10.- B. humidicola, 11.- Leucaena leucocephala, 12.- Macroptilium atropurpureum, 13.- Stylosanthes leiocarpa, 14.- $S$. capitata, 15.- C. pascuorum, 16.- C. arenarium, 17.- $S$. guianensis, 18.- Desmodium heterocarpon, 19.- S. macrocephala, 20.- D. canum, 21.- Arachis pintoi, 22. Pueraria phaseoloides, 23.- D. ovalifolium, 24.- Panicum maximum. 


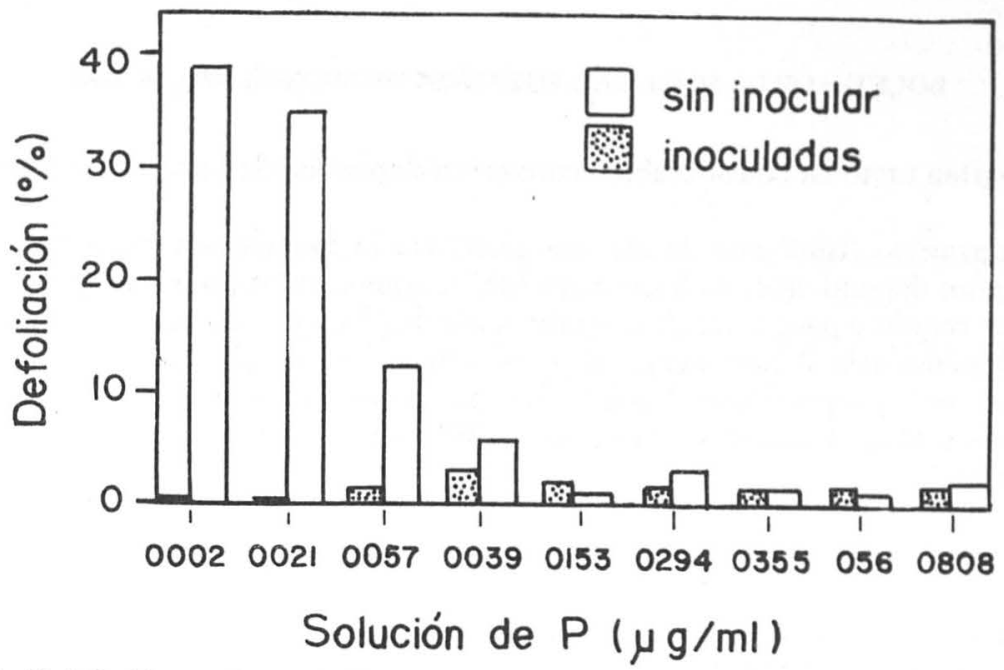

Figura 4.- Defoliación en plantas de Leucaena leucocephala micorrizadas y no micorrizadas creciendo a diferentes niveles de $\mathbf{P}$ en la solución de suelo.

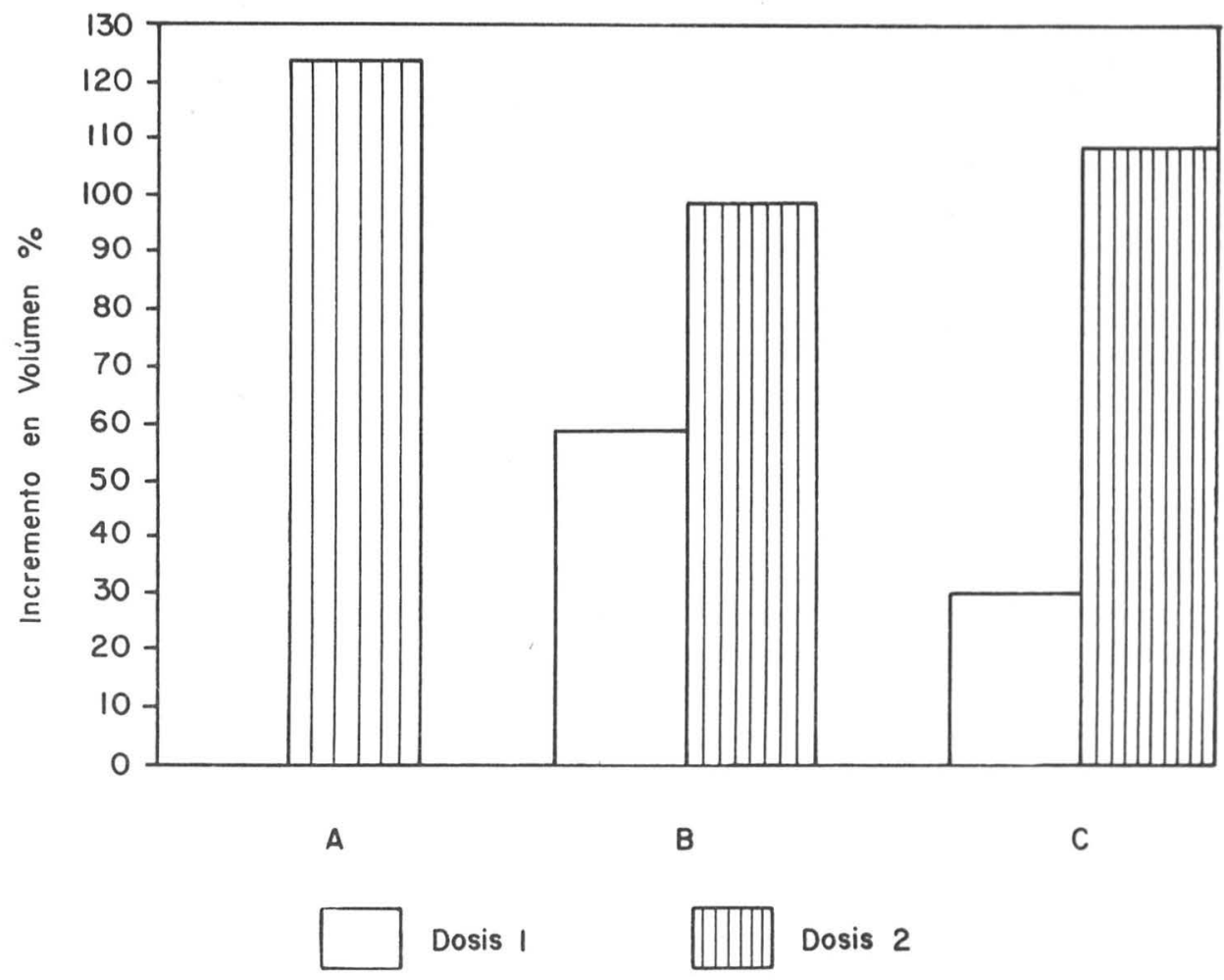

Figura 5.- Efecto de la inoculación con dos dosis de esporas de 3 diferentes hongos ectomicorrícicos en el incremento del volumen de plántulas de Pinus radiata, después de 25 semanas en vivero, en relación a las plántulas testigo (con micorrización espontánea). A.- Helvella lacunosa, B.- Russula brevipes, C.- Licoperdun perlatum. 
plantas varían también considerablemente en su dependencia micorrícica (Saif, 1987) (Fig.3).

La respuesta fisiológica de algunas plantas a la inoculación micorrícica puede mostrar gran dependencia de los hongos MVA, como Leucaena leucocephala que sin micorrizar requiere para su máximo rendimiento de 27 a 38 veces más de $\mathrm{P}$, disminuyendo esta dependencia al incrementar el contenido de $\mathrm{P}$ en la solución del suelo. En esta planta incluso la proporción de hojas tiradas por la misma es mucho mayor en ausencia de micorriza (Fig. 4 Habte y Manjunath, 1987) a bajas concentraciones del mismo nutriente.

c) Hongos.

Casi todos los suelos contienen esporas de hongos micorrícicos ó micorrizas formados por ellos. Sin embargo, no todos los hongos aportan el mismo grado de beneficio a las plantas (Shubert y Hayman, 1986; Valdés, 1985. Abbott y Robson, 1981). Existen diferencias muy marcadas en cuanto a la efectividad de los hongos micorrícicos en incrementar el crecimiento de las plantas. No hay evidencias de que exista una bien definida especifidad hospedera como existe en la asociación Rhizobium Leguminosa.

Los diferentes mecanismos fisiológicos dirigidos cada uno por genes específicos son indudablemente los que intervienen en el éxito de la interacción entre los hongos y las respuestas de la planta.

Los siguientes datos (Fig. 5) de ensayos con hongos ectomicorrícicos y con diferentes pinos, son un ejemplo de las bondades del uso de la micorriza y de la selección de los hongos en la producción de plántulas sanas y vigorosas en vivero, así como su posterior sobrevivencia y crecimiento al ser transplantadas a condiciones totalmente adversas, es decir transplantadas a un área totalmente erosionada donde, incluso se tuvo que recurrir a maquinaria pesada para hacer terrazas. La inoculación se hizo individualmente con micelio puro de uno de 3 diferentes hongos (Valdés, 1985).

No sólo se logran efectos positivos de inoculación con el uso de cultivos miceliales puros. el mismo beneficio se puede obtener con inoculación con esporas (Marx, et al., 1984.)

Esta última técnica es mucho más sencilla, económica y al alcance de todo vivero por modesto que sea. Basta con colectar carpóforos e inocular con ellos el suelo de los envases donde crecerán las plántulas (Tabla 1). 


\section{LITERATURA CITADA}

ABBOT, L. K. y A. D. RONSON 1981. Infectivity and effectiveness of five endomycorrhizal fungi: competition with indigenous fungi in field soils. Austr. J. Agric. Res. 32: 621-630.

AZCON, R. A., D. MARTIN y J. M. BAREA, 1978. Comparative role of phosphate in soil or in the host on the formation and effects and endomycorrhiza. Plant Soil 49: 561-567.

BAYLIS, G. T. S. 1970. Root hairs and phycomycetous mycorrhizas in phosphate-deficient soil. Plant Soil 33: 713-716.

BOWEN, G. D., M. F. SKINNER y D. I. BEVEGE, 1974. Zinc uptake by mycorrhizal and uninfected roots of Pinus radiata and Araucaria cunninghamii. Soil Biol. Biochem. 6: 141-144.

BOWEN, G. D., D. I. BEVEGE y B. MOSSE, 1975. The phosphate physiology of vesicular-arbuscular mycorrhizas. pp. 241-260. En: Sanders, F. E., B. Moose y P. B. Tinker (Edrs.). Endomycorrhizas. Academic Press, New York.

FITTER, A. H. 1985. Functioning of vesicular-arbuscular mycorrhizas under field conditions. New phytol. 99:: 257-260.

FURLAN, V. y J. A. FORTIN. 1973. Formation of endomycorrhizae by Endogoen calospora on Allium cepa under three temperature regimes. Nat. Canad. 100: 467-477.

FURLAN, V.y J. A. FORTIN, 1977. Effect of light intensity on the formation of VA mycorrhizas on Allium cepa by Gigaspora calospora. New Phytol. 79: 335-340.

GERDEMANN, J. W. 1975. Vesicular-arbuscular mycorrhizae. pp. 575-591. En: Torrey, J. G. y D. T. Clarkson. (Edrs.). The development and function of roots. Academic Press, New York.

HABTE, M. y A. MANJUNATH. 1987. Soil solution phosphorus status and mycorrhizal dependency in Leucaena leucocephala. App. Environ. Microbiol. 53: 797-801.

HAYMAN, D. S. 1970. Endogone spore numbers in soil and VAM in wheat as influenced by season and soil treatmeant. Trans. Brit. Mycol. Soc. 54: 53-63.

HAYMAN, D. S. 1982. Influence of soils and fertility on activity and survival of V. A. mycorrhizal fungi. Phytopathology 72: 1119-1125.

ITOH, S. y S. A. BARBER. 1983. Phosphorus uptake by six plants species as related to root hairs. Agron. J. $75: 457-461$.

MARX, D. H., K. JARL, J. RUEHLE y W. BELL. 1984. Development of Pisolithus tinctorius ectomycorrhizae of pine seedlings using basidiospore-encapsulated seeds. Forest Sci. 30: 897-907

MILLER, J. C., S. RAJAPAKSE y R. K. GARBER. 1986. Vesicular-arbuscular mycorrhizae in vegetable. Crop Hort. Science 21: 974-984.

NELSEN, C. E., N. C. BOLGIANO, S. C. FURUTANI, G. R. SAFIR y B. H. ZANDSTRA, 1981. The effect of soil phosphorus levels on mycorrhizal infection of field grown onion plants and on mycorrhizal reproduction. J. Amer. Soc. Hort. Sci. 106: 786-788.

NYE, P. B. 1977. The rate limiting step in plant nutrient absorption from soil. Soil Sci. 123: 292-297.

PLENCHETTE, C., J. A. FORTIN y V. FURLAN. 1983. Growth response of several plants species to mycorrhiza in a soil of moderate P-fertility. Plant Soil 70: 199-209. 
RHODES, L. H. y J. W. GERDEMANN, 1975. Phosphate uptake zones of mycorrhizal and non-mycorrhizal onions. New Phytol. 75: 555-561.

SAFIR, G. R. y C. E. NELSEN. 1980. Water and nutrient uptake by vesicular-arbuscular mycorrhizal plants. pp. 25-29. En: Myers, R. (Edr.). Role of mycorrhizal associations in crop productions. Proceedings of colloquial of the Rutgers University, New Brunswick, N. J.

SAIF, S. R. 1987. Growth responses of tropical forage plant species to vesicular-arbuscular mycorrhizae. Plant Soil 97: 25-35.

SCHUBERT, A. y D. S. HAYMAN, 1986. Plant growth responses to VAM, XVI. Effectiveness of different levels of soil phosphate. New Phytol. 103: 79-90.

VALDES, M. 1985. Survival and growth of pines with specific ectomycorrhizae after 3 years on a highly eroded site. Canad. J. Bot. $64:$ 885-888. 